\title{
ISRU Production of Life Support Consumables for a Lunar Base
}

\author{
Bonnie L. Cooper, Ph.D. \\ Oceaneering Space Systems \\ Tom Simon \\ NASA Johnson Space Center
}

\begin{abstract}
Similar to finding a home on Earth, location is important when selecting where to set up an exploration outpost. Essential considerations for comparing potential lunar outpost locations include: (1) areas nearby that would be useful for In-Situ Resource Utilization (ISRU) oxygen extraction from regolith for crew breathing oxygen as well as other potential uses; (2) proximity to a suitable landing site; (3) availability of sunlight; (4) capability for line-of-sight communications with Earth; (5) proximity to permanently-shadowed areas for potential in-situ water ice; and (6) scientific interest. The Mons Malapert ${ }^{1}$ (Malapert Mountain) area $\left(85.5^{\circ} \mathrm{S}, 0^{\circ} \mathrm{E}\right)$ has been compared to these criteria, and appears to be a suitable location for a lunar outpost.
\end{abstract}

\section{INTRODUCTION}

NASA is preparing the Constellation architecture to meet the exploration objective of developing a lunar outpost. The outpost location should be consistent with the architecture objectives, including future (Mars) exploration objectives. Many of the objectives for the outpost have already been defined. They include performing science, learning to survive on another planetary body, and learning to deal with the logistics issues of extended stays outside low Earth orbit. There are six criteria that should be considered for comparing potential lunar outpost sites: (1) having areas nearby that would be useful for ISRU oxygen production, (2) proximity to a suitable landing site, (3) availability of sunlight, (4) capability for line-of-sight communications with Earth, (5) proximity to permanently-shadowed areas for potential in-situ water, and (6) scientific interest. The Mons Malapert area is a suitable location for a lunar outpost, as described in the following sections.

\footnotetext{
${ }^{1}$ This name is unofficial; it has not been approved by the International Astronomical Union.
}

\section{PRODUCTION OF OXYGEN FOR CREW BREATHING AND OTHER USES}

Over twenty methods have been proposed for extracting oxygen from lunar regolith. Several methods have been demonstrated in laboratory-scale experiments on Earth and some are similar to processes used in industry on a larger scale. Three specific technologies are being pursued by NASA under the Exploration Technology Development Program (ETDP) project: hydrogen reduction, carbothermal reduction, and electrowinning. Hydrogen reduction has been demonstrated using Apollo samples. For this study we considered hydrogen reduction; however, similar metrics may be used when some other methods for extracting oxygen are considered.

The oxygen extraction process that is ultimately chosen will depend upon how well the overall system meets the requirements for the Constellation program. The ISRU system mass, including all power and excavation systems, must be less than the oxygen producedotherwise, it is a net loss to the architecture. Moreover, in order to provide the maximum benefit, ISRU must be planned during the architecture development rather than being added on after the architecture is set. Early planning allows ISRU to make a more significant impact on minimizing the upmass requirements, both during initial deployments as well as over the long term.

In the hydrogen reduction process, heated hydrogen will induce chemical reduction of the iron-bearing silicates in lunar regolith, which consist of ilmenite, olivine, pyroxene, and glass. The process produces water, which may be electrolyzed to obtain oxygen, and the hydrogen may then be recycled. A reactor for this process must be optimized to reduce the amount of electric power required while concurrently increasing oxygen yield. These competing requirements are partially answered by selecting a favorable feedstock for 


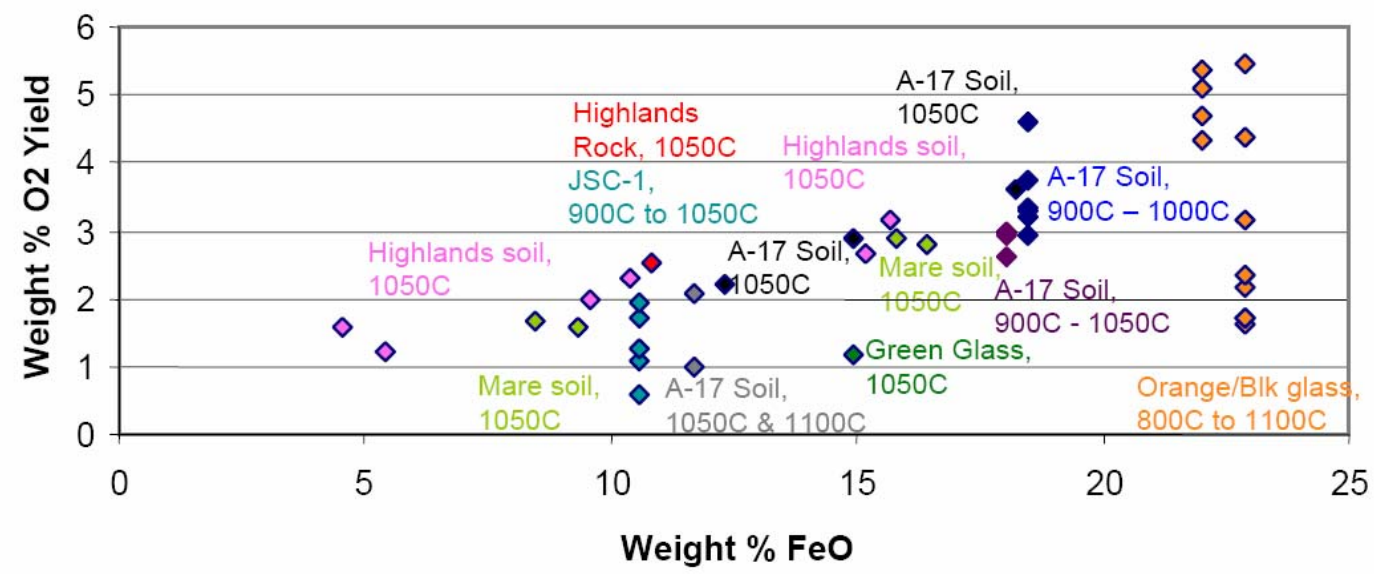

Figure 1. Weight percent of Iron Oxide (FeO) in a lunar soil directly affects the amount of oxygen (O2) that can be extracted from it. Published data on the amount of oxygen extracted from various types of soils using the hydrogen reduction process are shown here. Although the tests on these samples were performed at varying temperatures and for varying lengths of time, a trend can be seen that relates oxygen yield (wt\% O2 yield) to iron content (wt\% FeO). Data from [1].

the process. It has been shown by [2] that the amount of oxygen which can be recovered at a given location depends on the local chemistry and mineralogy of the soil. Specifically, the amount of oxygen that can be extracted by the hydrogen reduction process is directly correlated to the amount of iron in the sample (Figure 1) and the temperature at which the furnace is held.

Recent experimental efforts have included the testing of fluidized beds to compare the reaction rate and oxygen yield to laboratory pathfinder efforts (Figure 2). The experiments were performed assuming a polar location, where highlands regolith is predominant. Using highlands material (15 wt $\% \mathrm{FeO}$ or less, as shown in Figure 1), the maximum theoretical yield for a hydrogen reduction system would be approximately $1.6 \mathrm{wt} \%$ oxygen when operating the furnace at $1050^{\circ} \mathrm{C}$.

The entire south polar region was previously classified as anorthositic (low in iron and high in aluminum) compared to mare areas (which are typically at lower latitudes on the lunar near side). The possibility of finding more iron-rich materials in the polar regions is of interest from the standpoint of oxygen production as well as from the standpoint of scientific knowledge.

Recent studies have shown that there are patches of soil near the south pole that appear to have enhanced iron content [2]. The area surrounding Malapert Mountain was examined using the technique of [2], with positive results. There appears to be an area near the northeast base of Malapert Mountain which is richer in iron than other areas in the region (Figure 3 ).

\section{PROXIMITY TO A SUITABLE LANDING SITE}

A large $\left(165 \mathrm{~km}^{2}\right)$ relatively flat plain at the base of the mountain (approximately $25 \mathrm{~km}$ from the peak) provides a landing site for robotic or piloted
Figure 2. Test results for our first prototype showed a calculated oxygen yield of up to $1.25 \%$ based on Mößbauer (Mössbauer) analysis [4]. Yield improves with increased temperature, as shown by comparing sample \#9 (held at $997^{\circ} \mathrm{C}$ ) with sample \#11 (held at $851^{\circ} \mathrm{C}$ ). 


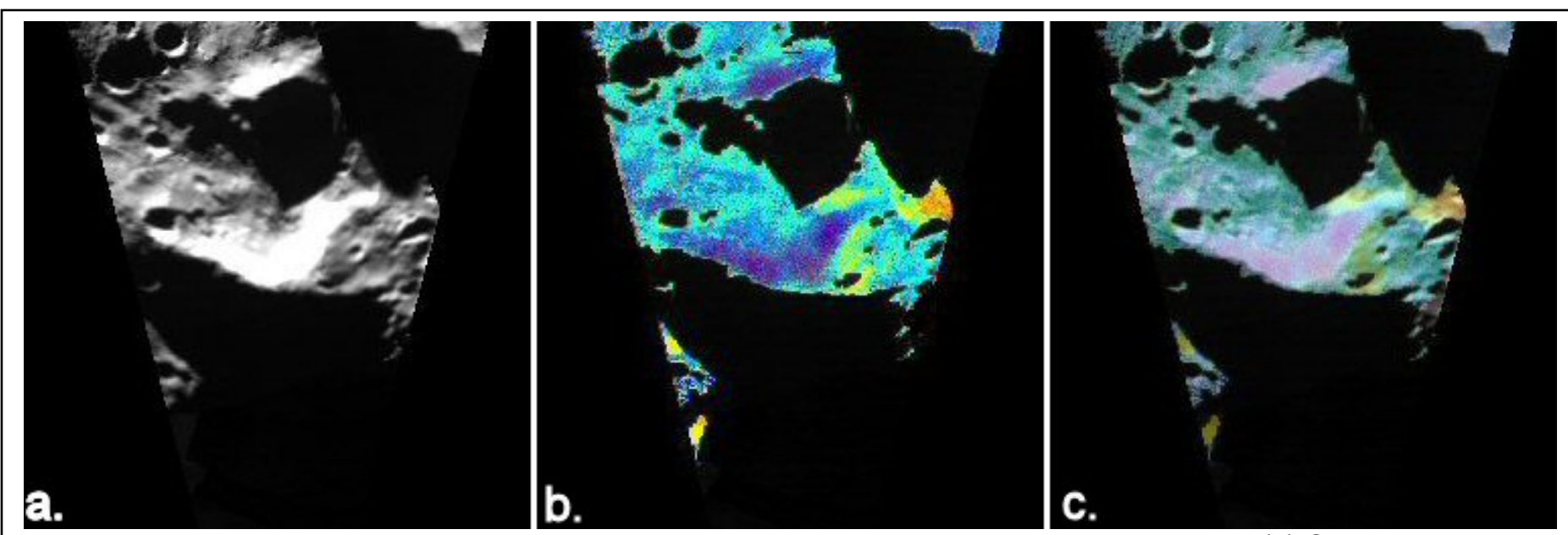

Figure 3. Areas near Mons Malapert that may have enriched iron content are shown in yellow. (a) Clementine $750 \mathrm{~nm}$ monochrome image of area. The peak of Mons Malapert is in the center of the image. (b) Clementine color ratio image of the same area, showing the possible iron-rich locations on the eastern flank. (c) Overlay of the two images shown in (a) and (b) which provides both topography and chemical data in one view.

missions. Furthermore, the slopes of the mountain range from six to thirty degrees. The gentler-gradient areas (from 6 to 12 degrees) should be navigable by a small rover in $1 / 6 \mathrm{~g}$ [4].

\section{AVAILABILITY OF SUNLIGHT}

Trade studies have demonstrated that the mass of the energy source dominates all other factors in determining the benefits of ISRU oxygen. Every process demonstrated to date requires a significant amount of energy, usually a mix of thermal and electrical energy. The availability of sunlight allows for the use of solar concentrators or other solar-energy-based methods to heat regolith. The ISRU system must produce a greater mass of oxygen than the mass of the production system itself, including the rovers for extracting and hauling feedstock, the power system, and other components. Utilizing solar energy is enabling for hydrogen reduction (especially at the poles) compared to more traditional energy sources that are being considered for the lunar outpost. Increased availability of sunlight results in lower mass for the ISRU system.

The summit of Malapert Mountain receives full or partial sunlight for $93 \%$ of the lunar year [6]. This makes it possible to minimize the mass of the night time power system and increase the use of solar concentrators for much of the work of heating the regolith. The higher the percentage of the year that there is sunlight available, the more time during the year oxygen can be produced. Accordingly, less mass would be needed for the system to produce an equivalent amount of oxygen.

\section{LINE-OF-SIGHT COMMUNICATIONS WITH EARTH}

Line-of-sight communications with the oxygen production plant (which must be operated robotically) is valuable because it reduces the amount of autonomy that is required of the system. Mobile robots will be used to transport feedstock to the plant and transfer the spent regolith to a designated location.

Maintaining constant communication with Earth is also beneficial in a more general sense for an early lunar base. Risk is reduced if the base has telemedicine capability such that flight surgeons can obtain timely information about any sickness or injury that might occur to a crew member. Furthermore, coordinating the efforts of the crew with ground control staff has proven useful throughout NASA's history with Apollo, Skylab, Shuttle, and ISS. It gives the crew access to real-time expertise for both contingencies as well as scheduled activities. At Mons Malapert, direct communications with Earth are possible at all times because Earth is always within view from Malapert Peak.

\section{PROXIMITY TO PERMANENTLY-SHADOWED AREAS}

The S-band radar data produced by [7] have been incorporated into a digital elevation model of the south polar region [8]. Previous workers [9] used digital elevation modeling to determine the locations of permanently-shadowed areas in the south pole region, which may contain water ice in useful quantities [10; 11]. Malapert Mountain is centrally located within $40 \mathrm{~km}$ of several of these large permanently-shadowed areas. A smaller permanently-shadowed region (about $4.5 \mathrm{~km}$ in area) is in a crater near the peak of Mons Malapert. Permanently-shadowed craters are of interest for gathering in-situ resources such as water ice, and also for gaining scientific knowledge about the ratio of water ice to other volatiles in cold-trap areas. Comparing these two permanently-shadowed regions would 


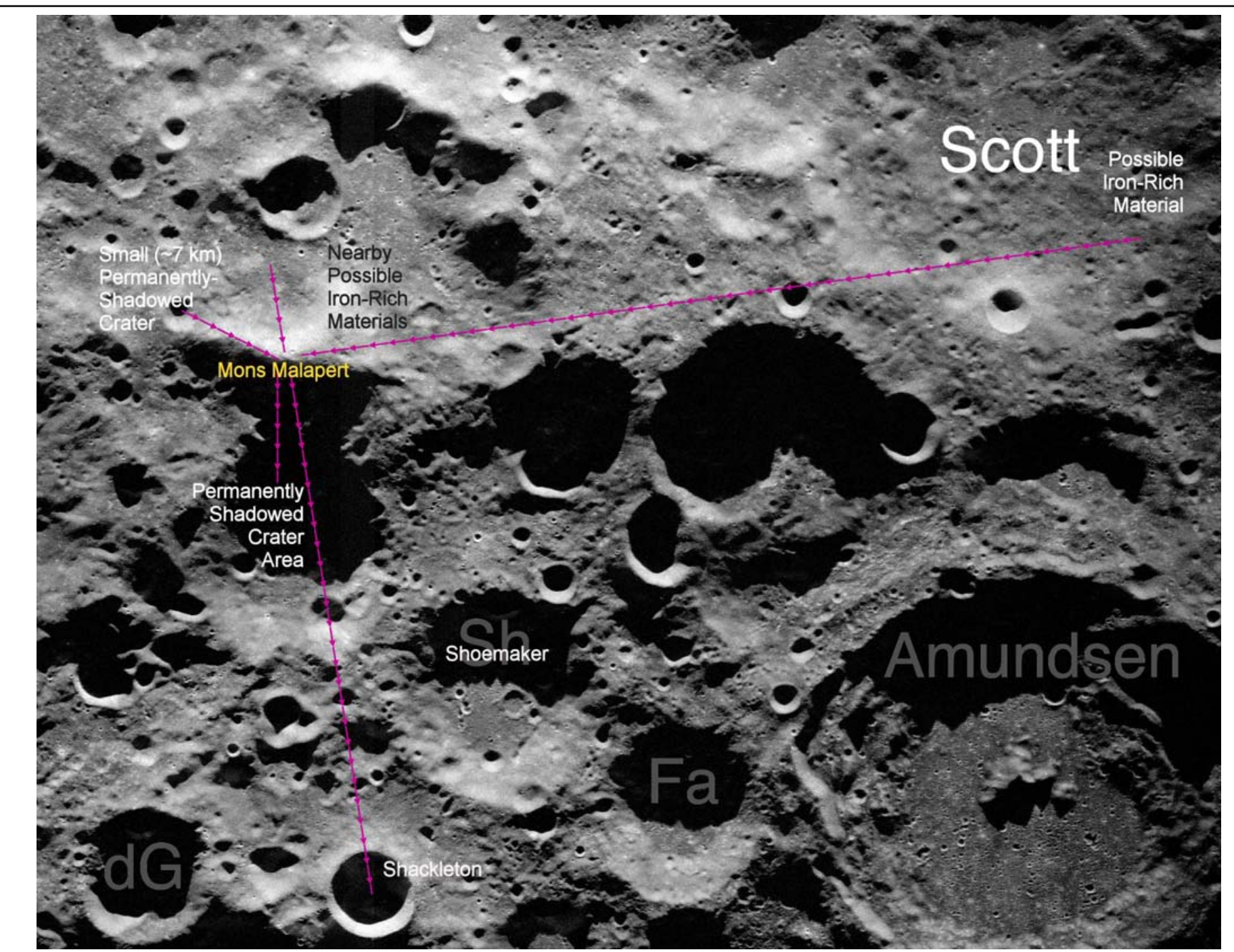

Figure 4. Map of lunar south polar region, showing Mons Malapert and the areas around it which would be useful for resource extraction or landing sites. See text for discussion.

improve our understanding of how the temperature of the surrounding terrain affects the temperature within the permanently-shadowed crater.

\section{SCIENTIFIC INTEREST}

Examination of Lunar Orbiter, Clementine, and newlyreleased radar data reveals oblong craters and channels near the peak of Malapert Mountain, in an area which does not appear to have volcanic materials at the surface [4]. Similar features are also seen in other areas nearby. The origin of these features, and their possible relationship to subsurface water ice, is of scientific interest. The radar data also show textural differences between the area nearest the crater and the area that is more distant. An absence of surface scatterers in the 1to 50-cm-size range may cause surfaces to appear lighter-colored in radar data. However, the reason for such a change in radar response between the upper and lower slope of Mons Malapert would be speculative until further analysis is completed. Additional investigation is needed to understand the geology of this region.

\section{FORWARD WORK}

To continue work in the analysis of Mons Malapert, several additional data processing steps are needed. In addition to removing spurious signals from the Clementine data that are caused by shadows, image corrections are needed to reduce the effects of spurious signal caused by strongly-lit sun-facing slopes in the polar regions [e.g. 13]. As planning progresses for the lunar outpost, these methods will allow us to estimate the actual iron content of the areas on the flanks of Mons Malapert. These analysis techniques can be performed at other polar locations as well.

With a more accurate estimate of the iron content in the soil, assessments can be made of the amount of oxygen that can be extracted per kilogram of regolith feedstock. These data will be coupled to design refinements to 
Table 1. Distances to features near Mons Malapert (see Figure 4 for locations).

\begin{tabular}{|l|c|c|}
\hline Feature & Distance, km & Distance, miles \\
\hline $\begin{array}{l}\text { Landing Site } \\
\text { (Flat area due north of Mons Malapert) }\end{array}$ & 17 & 11 \\
\hline $\begin{array}{l}\text { Closest Permanently-Shadowed Crater } \\
\text { (7-km-diameter crater on the flank of Mons Malapert) }\end{array}$ & 30 & 19 \\
\hline $\begin{array}{l}\text { Next-closest Permanently-Shadowed Crater } \\
\text { (Un-named crater due south of Mons Malapert) }\end{array}$ & 25 & 16 \\
\hline Possible Hi-Iron Deposits in Scott Crater & 211 & 131 \\
\hline Center of Shackleton Crater & 132 & 82 \\
\hline
\end{tabular}

determine the overall reduction in Earth launch mass (mass payback) for the Constellation architecture.

\section{CONCLUSIONS}

Based on the criteria presented here, the Mons Malapert site is well suited for the production of oxygen for life support. The potential higher iron content in some of the lunar regolith might enable a lower-mass oxygen extraction plant for the chosen process. Other benefits of this location include its proximity to a landing site; the long duration of sunlight per year (allowing for longer annual extraction operations); and continuous communication with Earth for telerobotic operations. Proximity to permanently-shadowed areas allow exploration for water ice which would be a useful resource. Finally, the convergence of engineering benefits and scientific interests at this location make it an attractive location for all stakeholders.

\section{COPYRIGHT}

This work is the property of the U.S. Government and as such is not subject to copyright.

\section{REFERENCES}

1. Allen, C.C.; Morris, R.V.; and McKay, D.S. "Experimental reduction of lunar mare soil and volcanic glass," Journal of Geophysical Research, Vol. 99, No. E-11, pp. 23,173-23,185, 1994.

2. Allen, C.C.; Knudsen, C. W.; Kanamori, H.; Morris, R. V.; Keller, L. P.; and McKay, D. S. "Lunar Resources - Oxygen from Rocks and Soil," In Workshop on Geology of the Apollo 17 Landing Site, pp. 1-2, Lunar Science Inst, Houston, Texas, 1992.

3. Cooper, B.L., "Mafic Materials in Scott Crater? A Test for Lunar Reconnaissance Orbiter," 38th Lunar and Planetary Science Conference, (Lunar and Planetary Science XXXVIII). LPI Contribution No. 1338, p.1377, 2007.

4. Morris, R. V.; Agresti, D. G.; Shelfer, T. D.; Pimperl, M. M.; Shen, M.-H.; Gibson, M. A.; Wills, E. L. "Moessbauer spectroscopy for lunar resource assessment: Measurement of mineralogy and soil maturity," In Lunar and Planetary Inst., Joint Workshop on New Technologies for Lunar Resource Assessment p 39-40, 1992.

5. Yoshida, K; Hamano, $\mathrm{H}$,; and Watanabe, $\mathrm{T}$. "SlipBased Traction Control of a Planetary Rover," Tohoku University and Mazda Motor Corporation, Sendai, Japan, ISER 2002.

6. Sharpe, Burton L.; Schrunk, David G. "Malapert mountain: Gateway to the moon," Advances in Space Research, Volume 31, Issue 11, p. 24672472, 2003.

7. Campbell, D.B.; Campbell, B.A., Carter, L.M.; Margo, J-L.; and Stacy, N.J.S. "No evidence for thick deposits of ice at the lunar south pole," Nature, Vol. 443 No. 7113, pp. 835-837, 2006.

8. Hare, Trent, personal communication, 2007.

9. Margot, J-L, "Topography of the Lunar Poles from Radar Interferometry: A Survey of Cold Trap Locations," Science, Vol. 284. no. 5420, pp. 1658 1660, 1999.

10. Nozette, S.; Spudis, P.D.; Robinson, M.S.; Bussey, D.B.J.; Lichtenberg, C.; and Bonner, R. "Integration of lunar polar remote-sensing data sets: Evidence for ice at the lunar south pole," Journal of Geophysical Research, Volume 106, Issue E10, p. 23253-23266, 2001.

11. Feldman, W.C.; Maurice, S.; Lawrence, D.J.; Little, R.C.; Lawson, S.L.; Gasnault, O.; Wiens, R.C.; Barraclough, B.L.; Elphic, R.C.; Prettyman, T.H.; Steinberg, J.T.; and Binder, A.B. "Evidence for Water Ice Near the Lunar Poles," Journal of Geophysical Research, Vol. 106, no. E10, pp. 23231-23251. 25 Oct. 2001

12. Cooper, B.L. "Craters and Channels On Malapert Mountain in The Lunar South Pole Region: Challenges Associated with High-Incidence-Angle Imagery," Space Resources Roundtable VIII, 2006.

13. Cahill, J.T.; Lucey, P.G.; and LeMouelic, S. "Apollo 17 landing site: evaluating a joint UVVIS-NIR FeO algorithm that nullifies topographic shading effects on lunar reflectance spectra," Lunar and Planetary Science XXXVIII, No. 1967, 2007.

\section{CONTACT}


Author contact information: bcooper@oceaneering.com 